\title{
A Novel Anti-jamming ADS-B Receiver Design Based on Wavelet Denoising Algorithm
}

\author{
Zhiyuan Shen ${ }^{+}$, Siyao Zhou and Hui Yu \\ College of Civil Aviation, Nanjing University of Aeronautics and Astronautics, China
}

\begin{abstract}
As a core technics for next generation air traffic control system, automatic dependent surveillance-broadcast (ADS-B) features faster updating rate and lower cost when comparing with secondary surveillance radar (SSR). The state-of-the-art ADS-B receiver instruments are constraint with multiple interferences problem. In this work, a universal software radio peripheral (USRP) based ADS-B receiver which is able to suppress several jamming interferences is achieved. The I/Q ADS-B signal was firstly implemented to wavelet based filter to suppress including out-of-channel blocking interference and inchannel pulse interference. Then a correlation algorithm was used to find real ADS-B digit report. A series of simulations and real-time experiments are used to verify the effectiveness of our proposed ADS-B receiver instrument.
\end{abstract}

Keywords: air traffic surveillance, automatic dependent surveillance-broadcast, anti-jamming, wavelet

\section{Introduction}

With the rapid growth of the global economy and travel demand, air traffic load experienced a tremendous growth. The conflict between the limited airspace resources and the increasing air traffic flow becomes more prominent. Automatic dependent surveillance-broadcast ADS-B technology [1] gets more precise target location and height information, which breaks traditional radar surveillance systems cannot cover the desert and ocean areas, in which the integrity and accuracy of data was limited [2]. The Single European Sky Programme (SESAR) by Euro Control and the Next Generation Air Transportation System (NextGen) by FAA are both considered ADS-Bas one of the key underlying technologies [3-4]. Compared to traditional data derived from ATC surveillance radar, ADS-B receives global navigation satellite system (GNSS) signals to provide accurate location information for air traffic controllers and pilots in the air. The aircraft airborne equipment combines the GNSS signal with the other relevant flight data and broadcasts them to other aircraft and air traffic management facilities [5].

As an open surveillance means, security issues of ADS-B becomes the biggest obstacle. The most prominent problem in ADS-B surveillance system security issues is man-made active jamming [6-7]. Jamming is generally with wide bandwidth. This kind of interference is able to detect, but hardly suppressed only by enhancing the anti-interference capability of receiving equipment. It causes that the ADS-B receiver cannot normally received position and velocity information of aircraft. This interference will mislead pilots and air traffic controllers and reduce the security level of the whole air traffic control automatic system. Therefore, interference problem is necessary to be solved for ADS-B system.

The state-of-the-art ADS-B receiver without anti-jamming ability cannot send / receive useful messages [8]. In this work, a universal software radio peripheral (USRP) based ADS-B receiver was achieved to suppress several jamming interferences.

\section{ADS-B Sampling}

\footnotetext{
+ Corresponding author. Tel.: +86-25-84893461; fax: +86-25-84893461.

E-mail address: shenzy@nuaa.edu.cn.
} 
USRP B210 platform provides a fully integrated signal processing ability which covers a continuous frequency range from $70 \mathrm{MHz}-6 \mathrm{GHzat}$ a single board. Designed for low cost experimental, it provides a real-time AD9361 RF integrated circuit transceiver with $56 \mathrm{MHz}$ bandwidth and a programmable Spartan6 FPGA with high speed USB 3.0 interface. And the USRP B210 platform with antenna is shown in Fig. 1.

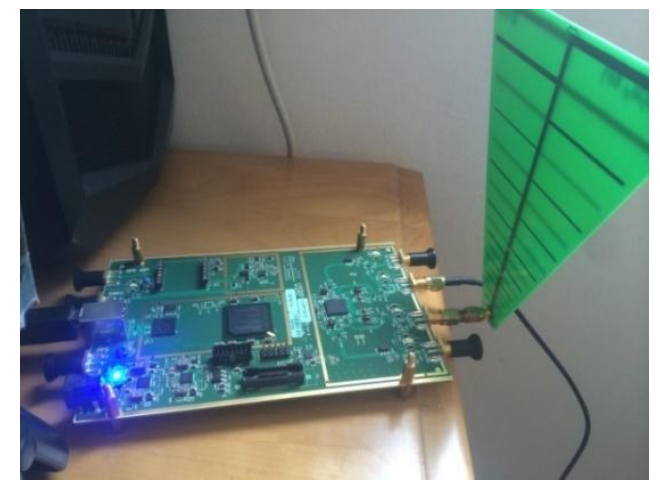

Fig. 1: USRP hardware platform.

The sampled ADS-B signal is modulated to a couple complex baseband signal (I and Q) by the RX local oscillator. Then the modulated I/Q signal are filtered by an analogy filter and sampled to digital signals. The final signals are preceded by PC machine. Due to PPM modulation based ADS-B information carried by the signal pulse position bearing, the phases of IQ signal does not include any useful information. Therefore, the envelope of digital complex signal is firstly extracted. Then they were synchronous and code decision according to the position information of the envelope. After the signal envelope is modulated to a series of digital sequences, we explore the corresponding ADS signal waveform head position and decoding to 0-1 data. Because ADS-B report is burst mode, the data is required to be synchronized. We note that each ADSB report is with a fixed $8 \mu$ s header format. So a local header template is set to correlation peak detection to determine the starting position of the message. The correlation between the receiver signal and the local template signal is calculated. The output waveform can be shown at the bottom of Fig. 3. It was found that a correlation peak existed at the start of the message, and the adjacent interval does not have larger peak output. Itis realized as a criterion that a starting point for real ADS-B message may be marked. Then according to the marked position of the received pulse, the following digit reports 0 s or $1 \mathrm{~s}$ are recorded. A CRC checking is implemented after decoding to eliminate the misjudgment influence caused by false peaks.

\section{Wavelet Based Filter Design}

\subsection{Category of Jamming}

Jamming usually includes sending malicious interference power to ADS-B receiver node (ground station or aircraft) at near frequency band of $1090 \mathrm{MHz}$.

Out-of-channel blocking interference is defined as the signal of which frequency bias to $1090 \pm 1 \mathrm{MHz}$. The offset frequency is usually twice larger than the channel width (2Mhz). It means that the frequency of interferences is usually set as $1095 \mathrm{MHz}$ or $1085 \mathrm{MHz}$. The power ratio between interference and the useful ADS-B signal is $20 \mathrm{~dB}$ or more. The peak of interference is higher $10 \mathrm{~dB}$ than that of ADS-B signal. For this kind of jamming interference, the lower frequency offset and stronger power will lead that the interference is closer to the channel and decrease the decoding rate for ADS-B receiver.

Another jamming is called as in-channel pulse interference. This kind of interference refers that the frequency of pulse signals are included by that of the receiver signal sampling bandwidth $(1089 \mathrm{MHz}$ to $1091 \mathrm{MHz})$. For pulse interference, the amplitude of pulse is usually lower than that of real signal. The stronger pulse power and longer pulse width will lead that the interference is closer to the channel and decrease the decoding rate for ADS-B receiver.

\subsection{Wavelet denoising algorithm}


To suppress jamming interference, a wavelet based denoising algorithm to achieve an adaptive high-pass and low-pass filter is proposed in this subsection.

For a received digit signal $\boldsymbol{x}(n)$, it is implemented to a conventional mlayer DB wavelet decomposition and reconstruction [9] as

$$
\boldsymbol{x}(n)=\sum_{k=1}^{K} \boldsymbol{x}_{k}(n)
$$

where $\boldsymbol{x}_{k}$ is $k^{\text {th }}$ reconstructed signal with wavelet coefficients.

The mean frequency of $x_{k}$ is usually estimated using the well-known autocorrelator by

$$
f_{k}=\frac{1}{2 \pi T_{s}}<\left[\frac{1}{N} \sum_{n=1}^{N-1} \boldsymbol{x}_{k}(n) \boldsymbol{x}_{k}^{*}(n-1)\right]
$$

where the notation $\angle$ denotes the angle of the complex term in the square bracket. Based on fundamental assumption that the frequency of out-of-channel blocking interference is not different $1090 \pm 1 \mathrm{MHz}$, a frequency threshold is firstly set to suppress this jamming. The output can be expressed as:

$$
\mathrm{y}=\sum_{\mathrm{k},\left|\mathrm{f}_{\mathrm{k}}-1090\right|<\mathrm{f}_{\mathrm{th}}}^{\mathrm{K}} \mathrm{x}_{\mathrm{k}}
$$

Furthermore, to suppress in-channel pulse interference, the mutual correlation between the $k^{t h}$ reconstructed signal $\boldsymbol{x}_{k}$ and the previous mentioned local ADS-B report template is calculated as

$$
e_{k}=\frac{1}{N} \sum_{n=1}^{N} \boldsymbol{x}_{k}(n) \boldsymbol{x}_{T}^{*}(n)
$$

According to the pulse length of interference is different from that of ADS-B burst, a predetermined energy threshold is set. The final output of wavelet based filter can be

$$
\boldsymbol{y}=\sum_{k,\left|f_{k}-1090\right|<f_{t h}, e_{k}>e_{t h}} \boldsymbol{x}_{k}
$$

\section{Simulation and Experiment}

600 random ADS-B signals are generated within 1 second. The SNR is set to 20dB. The out-of-channel blocking interference is firstly added with the offset frequency offset $4 \mathrm{MHz}$ and $40 \mathrm{~dB}$ power, as shown in Fig. 2. After passing the proposed wavelet based filter, the correlation detection is implemented to find ADS-B signal. The results shows that 600 signals are corrected decoded and the detection Rate $=100.00 \%$.
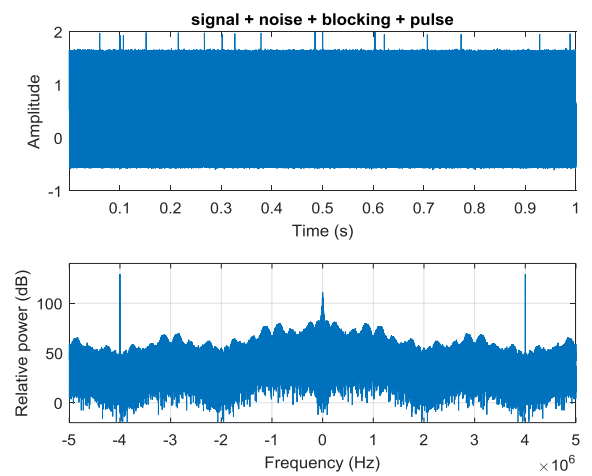

Fig. 2: 600 ADS-B signal with 600 in-channel pulse interference with $0.3 M \mu s$ pulse width at 1 second.

Then in-channel pulse interferences are added into above simulated signals. Same as the previous parameters setting, 600 random ADS-B signals are generated within 1 second with $\mathrm{SNR}=20 \mathrm{~dB}$. The offset 
frequency of out-of-channel wideband frequency interference is set as offset $4 \mathrm{MHz}$ with $40 \mathrm{~dB}$ power ratio between interference ADS-B signals. 600 added pulse interferences with twice amplitude than that of the signal and pulse width of $0.3 \mathrm{M \mu s}$ are originated. The time domain and domain frequency chart are shown in Fig. 3. According to filter and correlation algorithm, the correct decoding 600 ADS-B signals are found with detection rate $=100.00 \%$.

The in-channel pulse interferences are enhanced by setting 4000 pulse interference with 2.5 times amplitude than that of the signal and pulse width of $0.5 M \mu s$. The time domain and domain frequency chart are shown in Fig. 4.According to filter and correlation algorithm, the correct decoding 552ADS-B signals are found with detection rate $=92.00 \%$.
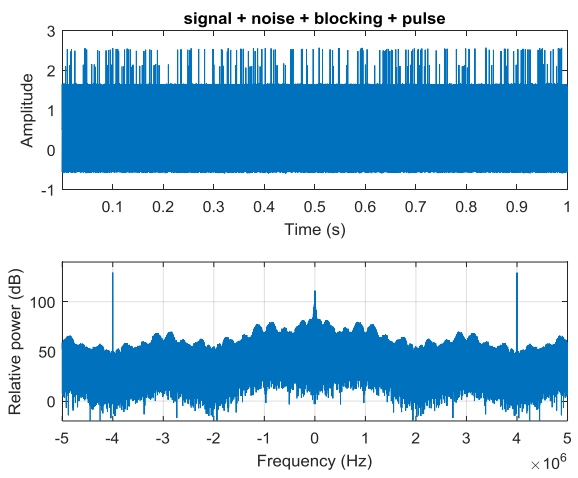

Fig. 3: 600 ADS-B signal with 4000 in-channel pulse interference with $0.5 \mathrm{M} \mu$ s pulse width at 1 second.

\section{Conclusion}

In this work, a universal software radio peripheral (USRP) based ADS-B receiver which is able to suppress several jamming interferences was achieved. The I/Q ADS-B signal was firstly implemented to wavelet based filter to suppress jamming including out-of-channel blocking interference and in-channel pulse interference. Then a correlation algorithm was used to find real ADS-B digit report. A series of simulations and real-time experiments are used to verify the effectiveness of our proposed ADS-B receiver instrument.

\section{Acknowledgements}

The authors would like to thank the financial support by National Natural Science Foundation of China (No.61501225)and Fundamental Research Funds for the Central Universities (NZ2015105 and NZ2016109).

\section{References}

[1] C. Rekkas,and M. Rees, “Towards ADS-B Implementation in Europe,"in Proc. International Workshop on Digital Communications-Enhanced Surveillance of Aircraft and Vehicles, Italy, September 3-5, 2008.

[2] H. Erzberger,and R. A. Paielli, “Concept for Next Generation Air Traffic Control System,” Air Traffic Control Quarterly, 2002, 10 (4): 355-378.

[3] D. McCallie, J. Butts, and R. Mills, "Security Analysis of the ADSB Implementation in the Next Generation Air Transportation System,’'International Journal of Critical Infrastructure Protection, 2011, 4 (2):78-87.

[4] M. Storhmeier, M. Schafer, and V. Lenders, "Realities and Challenges of NextGenAir Traffic Management: the Case of ADS-B," IEEE Communication Magazine, 2014, 52(5): 111-118.

[5] M. Schafer, M. Storhmeier, and V. Lenders, "Bringing up opensky: a large-scale ADS-B sensor network for research," in Proc.13th International Symposium on Information Processing in Sensor Networks, Berlin, Germany April 15-17, 2014.

[6] M. Storhmeier, V. Lenders, and I. Martinovic. "On the Security of the Automatic Dependent SurveillanceBroadcast Protocol,” IEEE Communications Surveys \& Tutorials, 2014, 17(2):1066-1087. 
[7] E. Chan-Tin, V. Heorhiadi, and N. Hopper, "The Frog-boiling Attack: Limitations of Secure Network Coordinate Systems,"ACM Transactions on Information and System Security, 2011, 14 (3):1-27.

[8] X Lin, J Zhang, and Y Zhu, "Simulation Study of Algorithms for Aircraft Trajectory Prediction based on ADS-B Technology,"in Proc. 7th International Conference on System Simulation and Scientific Computing, Asia Simulation Conference, 2008, pp. 322-327.

[9] P. Flandrin, G. Rilling, and P. Concalves, "Empirical Mode Decomposition as a Filter Bank," IEEE Signal Processing Letter, 2004, 11(2):112-114. 